\title{
Subordination Results Associated With Generalized Bessel Functions
}

\author{
Hamzat J. Olusegun \\ University of Lagos, \\ Akoka, Lagos State, Nigeria \\ Correspondence to: Hamzat J. Olusegun, Email: jhamzat@unilag.edu.ng
}

\begin{abstract}
In the present work, subordination results for function $f(z)^{\gamma}$ belonging to a new class of analytic function $S_{n}^{\gamma}(\alpha, \beta, j)$ defined using the concept of Hadamard product are obtained. Also coefficient estimates, growth and distortion properties for function $f(z)^{\gamma}$ in the class $R_{n}^{\gamma}(\alpha, \beta, j)$ of Bessel type are equally investigated.
\end{abstract}

Keywords: Analytic, Univalent, Bazilevic function, Bessel function, Growth and distortion bounds.

\section{Introduction and Preliminaries}

Denoted by A the usual class of analytic functions $f(z)$ having the form

$$
f(z)=z+\sum_{k=j+1}^{\infty} a_{k} z^{k} \quad j \in N
$$

normalised with $f(0)=f^{\prime}(0)-1=0$ in the unit disk $U=\{z \in \mathbb{C}:|z|<1\}$. Also, let $S \subset A$ denote the class of all analytic univalent functions in the open unit disk U. Suppose that an index $\gamma(\gamma$ is real) is posed on $f(z)$ defined in (1), then

$$
f(z)^{\gamma}=\left(z+\sum_{k=j+1}^{\infty} a_{k} z^{k}\right)^{\gamma}
$$

Expanding (2) binomially, we obtain

$$
f(z)^{\gamma}=z^{\gamma}+\sum_{k=j+1}^{\infty} a_{k}(\gamma) z^{\gamma+k-1}, \quad \gamma>0 .
$$

Now, for functions $f(z) \in A$, Salagean introduced and studied the following differential operator:

$$
\begin{gathered}
D^{0} f(z)=f(z) \\
D^{\prime} f(z)=D\left(D^{0} f(z)\right)=z f^{\prime}(z) \\
D^{n} f(z)=D\left(D^{n-1} f(z)\right)=z\left(D^{n-1} f(z)\right)^{\prime},
\end{gathered}
$$

(see $[2,8,9]$ ). Thus, with the aid of (3) and (4) we obtain the differential operator

$$
D^{n} f(z)^{\gamma}=\gamma^{n} z^{\gamma}+\sum_{k=j+1}^{\infty}(\gamma+k-1)^{n} a_{k}(\gamma) z^{\gamma+k-1} .
$$

In 1994, Opoola [9] studied a special class of analytic funtion denoted by $T_{n}^{\gamma}(\beta)$ satisfying the inequality

$$
\Re\left\{\frac{D^{n} f(z)^{\gamma}}{z^{\gamma}}\right\}>\beta, \quad z \in U
$$

and his study has led to the development of some properties of the class $T_{n}^{\gamma}(\beta)$. In the recent time, the class $T_{n}^{\gamma}(\beta)$ of Bazilevic type has gained the attention of both young and old researchers in the field of complex 
analysis (especially univalent function theory) and the result of their findings authenticated diversely in literatures (see [2,8] among others). However, a litle modification was made to (6) by Babalola [1] such that

$$
\Re\left\{\frac{D^{n} f(z)^{\gamma}}{\gamma^{n} z^{\gamma}}\right\}>\beta, \beta \geq 0, z \in U
$$

where $\gamma>0, n \in N_{0}=N \bigcup\{0\}$ and $D^{n}$ is the well-known Salagean derivative operator.

Here, we recall that the second-order linear homogeneous differential equation (that is the generalized Bessel differential equation)

$$
z^{2} w^{\prime \prime}(z)+b z w^{\prime}(z)+\left[c z^{2}-p^{2}+(1-b) p\right] w(z)=0
$$

has a particular solution $u_{p}(z)$ which has the series form

$$
u_{p}(z)=\sum_{k=0}^{\infty} \frac{(-c)^{k}}{k ! \Gamma\left(p+k+\frac{b+1}{2}\right)}\left(\frac{z}{2}\right)^{2 k+p} .
$$

The function $u_{p}(z)$ in $(9)$ is referred to as the generalized Bessel function of the first kind and of order $p$, where $b, c, p \in \mathbb{C}$.

Incidentally, $u_{p}(z)$ can be normalized using the transformation

$$
u_{p}(z)=\left[a_{0}(p)\right]^{-1} z^{-\frac{p}{2}} w_{p}\left(z^{\frac{1}{2}}\right)
$$

such that

$$
u_{p}(z)=\sum_{k=0}^{\infty} \frac{(-c / 4)^{k}}{(m)_{k} k !} z^{k}
$$

where $m=p+\frac{b+1}{2}$ and $z \in \mathbb{C}$ (see $\left.[4,7,10]\right)$. It is pertinent to note that the function defined in (11) is analytic and satisfies the second-order linear differential equation

$$
4 z^{2} u^{\prime \prime}(z)+2[2 p+b+1] z u^{\prime}(z)+c z u(z)=0 .
$$

From (11), one can write that

$$
z u_{p}(z)=z+\sum_{k=j+1}^{\infty} \frac{(-c / 4)^{k-1}}{(m)_{k-1}(k-1) !} z^{k}, \quad j \in N
$$

Using the concept of convolution (Hadamard product), we have that

$$
J_{n}^{\gamma}(\beta, j) f(z)^{\gamma}=D^{n} f(z)^{\gamma} * z u_{p}(z)=\gamma^{n} z^{\gamma}+\sum_{k=j+1}^{\infty}(\gamma+k-1)^{n} \psi_{k} a_{k}(\gamma) z^{\gamma+k-1}
$$

where

$$
\psi_{k}=\frac{(-c / 4)^{k-1}}{(m)_{k-1}(k-1) !}, \quad m=p+\frac{b+1}{2}, \text { and } b, c, p \in \mathbb{C} .
$$

Suppose that $\beta \geq 0,-1 \leq \alpha<1$, and $\gamma>0$. We let $S_{n}^{\gamma}(\alpha, \beta, j)$ denote the subclass of $S$ consisting of function $f(z)^{\gamma}$ of the form $(3)$ and satisfy the analytic criterion

$$
\operatorname{Re}\left\{\frac{J_{n}^{\gamma}(\beta, j) f(z)^{\gamma}}{\gamma^{n} z^{\gamma}}-\alpha\right\}>\beta\left|\frac{J_{n}^{\gamma}(\beta, j) f(z)^{\gamma}}{\gamma^{n} z^{\gamma}}-1\right| .
$$

Further, let

$$
R S_{n}^{\gamma}(\beta, j)=S_{n}^{\gamma}(\beta, j) \cap R
$$


where $R$ is the subclass of $S$ consisting of functions of the form

$$
f(z)^{\gamma}=z^{\gamma}-\sum_{k=j+1}^{\infty} a_{k}(\gamma) z^{\gamma+k-1}, \quad a_{k}(\gamma)>0, \quad \gamma>0, \text { and } j \in N
$$

The following lemmas shall be necessary for the purpose of our present investigations (see $[3,6]$ ).

Lemma 1.1. Let $\delta \neq 0$ be a complex number. Also let $q(z)$ be such that $q(z) \neq 0$ and a univalent function in $U$, we have that

$$
\operatorname{Re}\left\{1+\frac{z q^{\prime \prime}(z)}{q^{\prime}(z)}-\frac{z q^{\prime}(z)}{q(z)}\right\}>\max \left\{0, \operatorname{Re}\left(\frac{\delta-1}{\delta} q(z)\right)\right\} .
$$

If $p,(p(z) \neq 0), z \in U$ satisfies the differential equation

$$
(1-\delta)(p(z)-1)+\delta \frac{z p^{\prime}(z)}{p(z)} \prec(1-\delta)(q(z)-1)+\delta \frac{z q^{\prime}(z)}{q(z)}
$$

then $p \prec q$ and $q$ is the best dominant (see[12]).

Lemma 1.2. Let $\omega$ be analytic in $U$ with $\omega(0)=0$. If $|\omega(z)|$ attains its maximum value on the circle $|z|<1$ at a point $z_{0}$, then

$$
z_{0} \omega^{\prime}\left(z_{0}\right)=t \omega\left(z_{0}\right)
$$

where $t$ is a real number and $t \geq 1(\operatorname{see}[5,8])$.

Lemma 1.3. If $f \in A$ satisfies

$$
\operatorname{Re}\left[1+\frac{z f^{\prime \prime}(z)}{f^{\prime}(z)}\right]<\frac{\mu+1}{2(\mu-1)},(2 \leq \mu<3, z \in U)
$$

then

$$
\frac{z f^{\prime}(z)}{f(z)} \prec \frac{\mu(1-z)}{\mu-z}
$$

and

$$
\left|\frac{z f^{\prime}(z)}{f(z)}-\frac{\mu}{\mu+1}\right|<\frac{\mu}{\mu+1}
$$

$(\operatorname{see}[10])$.

\section{Subordination Results}

Theorem 2.4. Let $\gamma$ be real $(\gamma>0), n \in N_{0}=N \cup\{0\}$ and $\delta \neq 0$ be a complex number such that

$$
\operatorname{Re}\left\{1+\frac{z(1-t+2 t z)}{(1+t z)(1-z)}\right\}>\max \left\{0, \operatorname{Re}\left(\frac{\delta-1}{\delta}\right)\left(\frac{1+t z}{1-z}\right)^{\gamma}\right\} \text {. }
$$

If the subordination

$$
\begin{aligned}
(1-\delta) & {\left[\frac{z\left(J_{n}^{\gamma}(\beta, j) f(z)^{\gamma}\right)^{\prime}}{\gamma^{n+1} z^{\gamma}}-1\right]+\delta\left[1-\gamma+\frac{z\left(J_{n}^{\gamma}(\beta, j) f(z)^{\gamma}\right)^{\prime \prime}}{\left(J_{n}^{\gamma}(\beta, j) f(z)^{\gamma}\right)^{\prime}}\right] } \\
& \prec(1-\delta)\left[\left(\frac{1+t z}{1-z}\right)^{\gamma}-1\right]+\frac{\delta \gamma(1+t) z}{(1+t z)(1-z)}
\end{aligned}
$$


holds true. Then $f(z)^{\gamma} \in S_{n}^{\gamma}(\alpha, \beta, j)$.

Proof. Upon setting

$$
p(z)=\frac{z\left(J_{n}^{\gamma}(\beta, j) f(z)^{\gamma}\right)^{\prime}}{\gamma^{n+1} z^{\gamma}} \text { and } q(z)=\left(\frac{1+t z}{1-z}\right)^{\gamma}
$$

Then,

$$
\begin{gathered}
\operatorname{Re}\left\{1+\frac{z q^{\prime \prime}(z)}{q^{\prime}(z)}-\frac{z q^{\prime}(z)}{q(z)}\right\}>\max \left\{0, \operatorname{Re}\left(\frac{\delta-1}{\delta}\right)\left(\frac{1+t z}{1-z}\right)^{\gamma}\right\}=\max \left\{0, \operatorname{Re}\left(\frac{\delta-1}{\delta} q(z)\right)\right\} \\
(1-\delta)(p(z)-1)+\delta \frac{z p^{\prime}(z)}{p(z)}=(1-\delta)\left[\frac{z\left(J_{n}^{\gamma}(\beta, j) f(z)^{\gamma}\right)^{\prime}}{\gamma^{n+1} z^{\gamma}}-1\right]+\delta\left[1-\gamma+\frac{z\left(J_{n}^{\gamma}(\beta, j) f(z)^{\gamma}\right)^{\prime \prime}}{\left(J_{n}^{\gamma}(\beta, j) f(z)^{\gamma}\right)^{\prime}}\right] \\
\prec(1-\delta)\left[\left(\frac{1+t z}{1-z}\right)^{\gamma}-1\right]+\frac{\delta \gamma(1+t) z}{(1+t z)(1-z)}=(1-\delta)(q(z)-1)+\delta \frac{z q^{\prime}(z)}{q(z)}
\end{gathered}
$$

In view of Lemma 2.1, we have established that $f(z)^{\gamma} \in S_{n}^{\gamma}(\alpha, \beta, j)$ of Bessel type. If we let $\gamma=1, n=0$ and $t=1$, then the following Corollary is immediate.

Corollary 2.5. Let $\delta \neq 0$ be a complex number such that

$$
\operatorname{Re}\left\{1+\frac{2 z^{2}}{1-z^{2}}\right\}>\max \left\{0, \operatorname{Re}\left[\left(\frac{\delta-1}{\delta}\right)\left(\frac{1+z}{1-z}\right)\right]\right\} .
$$

If the subordination

$$
(1-\delta)\left[\left(J_{0}^{\prime}(\beta, j) f(z)^{\gamma}\right)^{\prime}-1\right]+\delta\left[\frac{z\left(J_{0}^{\prime}(\beta, j) f(z)^{\gamma}\right)^{\prime \prime}}{\left(J_{0}^{\prime}(\beta, j) f(z)^{\gamma}\right)^{\prime}}\right] \prec(1-\delta)\left[\frac{1+z}{1-z}-1\right]+\frac{2 \delta z}{1-z^{2}}
$$

holds true, then $f(z)^{\gamma}$ belongs to the class $S_{0}^{1}(\alpha, \beta, j)$.

Theorem 2.6. Let the function $f(z)^{\gamma}$ be of the form (16) and satisfying the condition that

$$
\operatorname{Re}\left\{1-\gamma+\frac{z\left(J_{n}^{\gamma}(\beta, j) f(z)^{\gamma}\right)^{\prime \prime}}{\left(J_{n}^{\gamma}(\beta, j) f(z)^{\gamma}\right)^{\prime}}\right\}<\frac{\gamma(t+1)}{2(t-1)} \quad t>1, \gamma>0, z \in U
$$

Then $f(z)^{\gamma}$ belong to the class $S_{n}^{\gamma}(\alpha, \beta, j)$ of Bessel type.

Proof. Let $\omega$ be defined by

$$
\frac{z\left(J_{n}^{\gamma}(\beta, j) f(z)^{\gamma}\right)}{\gamma^{n+1} z^{\gamma}}=\left(\frac{1+t \omega(z)}{1-\omega(z)}\right)^{\gamma}, \quad \omega(z) \neq 1
$$

Then, $\omega$ is analytic in $U$, and since $t \neq 1$, therefore $\omega(0)=0$. Also it follows that,

$$
\operatorname{Re}\left\{1-\gamma+\frac{z\left(J_{n}^{\gamma}(\beta, j) f(z)^{\gamma}\right)^{\prime \prime}}{\left(J_{n}^{\gamma}(\beta, j) f(z)^{\gamma}\right)^{\prime}}\right\}=\operatorname{Re}\left\{\frac{\gamma(t+1) z \omega^{\prime}(z)}{(1+t \omega(z))(1-\omega(z))}\right\}<\frac{\gamma(t+1)}{2(t-1)} \quad t \neq 1
$$

Next, we show that $|\omega(z)|<1$.

Let there exists a point $z_{0} \in U$ such that

$$
\underbrace{\max }_{|z| \leq\left|z_{0}\right|}|\omega(z)|=|\omega(z)|=1
$$

Then, using Lemma 2.3 and letting $\omega\left(z_{0}\right)=e^{i \theta}$ and $z_{0} \omega^{\prime}\left(z_{0}\right)=\sigma e^{i \theta}, \sigma \geq 1$, we obtain

$$
\operatorname{Re}\left\{1-\gamma+\frac{z\left(J_{n}^{\gamma}(\beta, j) f(z)^{\gamma}\right)^{\prime \prime}}{\left(J_{n}^{\gamma}(\beta, j) f(z)^{\gamma}\right)^{\prime}}\right\}=\operatorname{Re}\left\{\frac{\gamma(t+1) z \omega^{\prime}(z)}{(1+t \omega(z))(1-\omega(z))}\right\}
$$




$$
=\operatorname{Re}\left\{\frac{\gamma \sigma e^{i \theta}(t+1)}{\left(1+t e^{i \theta}\right)\left(1-e^{i \theta}\right)}\right\}=\frac{\gamma \sigma(t+1)}{2(t-1)} \geq \frac{\gamma(t+1)}{2(t-1)} t>1, \quad \gamma>0
$$

Thus,

$$
\operatorname{Re}\left\{1-\gamma+\frac{z\left(J_{n}^{\gamma}(\beta, j) f(z)^{\gamma}\right)^{\prime \prime}}{\left(J_{n}^{\gamma}(\beta, j) f(z)^{\gamma}\right)^{\prime}}\right\} \geq \frac{\gamma(t+1)}{2(t-1)}, \quad(z \in U)
$$

which counter the hypothesis (23). Therefore, we conclude by saying that $|\omega(z)|<1$ for all $z \in U$ and

$$
\frac{z\left(J_{n}^{\gamma}(\beta, j) f(z)^{\gamma}\right)}{\gamma^{n+1} z^{\gamma}} \prec\left(\frac{1+t z}{1-z}\right)^{\gamma}, \quad t \neq 1, \gamma>0, \quad z \in U
$$

and this completes the proof.

\section{Coefficient Estimates}

In this section, we present both the coefficient estimate results and the necessary and sufficient condition for function $f(z)^{\gamma}$ of the form (16) belonging to the class $R S_{n}^{\gamma}(\alpha, \beta, j)$ of Bessel type.

Theorem 3.1. A function $f(z)^{\gamma}$ having the form (16) belong to the class $R S_{n}^{\gamma}(\alpha, \beta, j)$ of Bessel type if for $\beta \geq 0,-1 \leq \alpha<1$ and $\gamma>0$

$$
\sum_{k=j+1}^{\infty}\left(\frac{\gamma+k-1}{\gamma}\right)^{n}\left|\psi_{k}\right|\left|a_{k}(\gamma)\right| \leq \frac{1-\alpha}{1+\beta}
$$

where

$$
\psi_{k}=\frac{(-c / 4)^{k-1}}{(m)_{k-1}(k-1) !}, \quad m=p+\frac{b+1}{2}
$$

where $b, c$ and $p$ belong to the set of complex numbers.

Proof. It is sufficient to show that

$$
\beta\left|\frac{J_{n}^{\gamma}(\beta, j) f(z)^{\gamma}}{\gamma^{n} z^{\gamma}}-1\right|-\operatorname{Re}\left\{\frac{J_{n}^{\gamma}(\beta, j) f(z)^{\gamma}}{\gamma^{n} z^{\gamma}}-1\right\} \leq 1-\alpha .
$$

It follows that

$$
\begin{aligned}
\beta\left|\frac{J_{n}^{\gamma}(\beta, j) f(z)^{\gamma}}{\gamma^{n} z^{\gamma}}-1\right|-\operatorname{Re}\left\{\frac{J_{n}^{\gamma}(\beta, j) f(z)^{\gamma}}{\gamma^{n} z^{\gamma}}-1\right\} \leq(1+\beta)\left|\frac{J_{n}^{\gamma}(\beta, j) f(z)^{\gamma}}{\gamma^{n} z^{\gamma}}-1\right| \\
=(1+\beta)\left|\sum_{k=j+1}^{\infty}\left(\frac{\gamma+k-1}{\gamma}\right)^{n} \psi_{k} a_{k}(\gamma)\right| .
\end{aligned}
$$

The last expression is bounded above by $1-\alpha$ if

$$
\sum_{k=j+1}^{\infty}(1+\beta)\left(\frac{\gamma+k-1}{\gamma}\right)^{n}\left|\psi_{k}\right|\left|a_{k}(\gamma)\right| \leq 1-\alpha .
$$

Hence the prove.

Corollary 3.2. A function $f(z)^{\gamma}$ having the form (16) belong to the class $R S_{n}^{1}(\alpha, \beta, j)$ of Bessel type if

$$
\sum_{k=j+1}^{\infty} k^{n}\left|\psi_{k}\right|\left|a_{k}\right| \leq \frac{1-\alpha}{1+\beta}, \quad a_{k}(1)=a_{k} .
$$


Corollary 3.3. A function $f(z)^{\gamma}$ having the form (16) belong to the class $R S_{n}^{1}(\alpha, 0, j)$ of Bessel type if

$$
\sum_{k=j+1}^{\infty} k^{n}\left|\psi_{k}\right|\left|a_{k}\right| \leq 1-\alpha
$$

Corollary 3.4. A function $f(z)^{\gamma}$ having the form (16) belong to the class $R S_{n}^{1}(\alpha, 0, j)$ of Bessel type if

$$
\sum_{k=j+1}^{\infty} k^{n}\left|\psi_{k}\right|\left|a_{k}\right| \leq 2
$$

Theorem 3.5. A necessary and sufficient condition for function $f(z)^{\gamma}$ of the form (16) to be in the class $R S_{n}^{\gamma}(\alpha, \beta, j)$ is that

$$
\begin{aligned}
& \sum_{k=j+1}^{\infty}(1+\beta)\left(\frac{\gamma+k-1}{\gamma}\right)^{n}\left|\psi_{k}\right|\left|a_{k}(\gamma)\right| \leq 1-\alpha \\
& \quad \gamma>0, \quad \beta \geq 0, \quad-1 \leq \alpha<1, \quad n \in N \cup\{0\} .
\end{aligned}
$$

Proof. In view of Theorem 3.1, we only need to prove the necessity. Now, if $f(z)^{\gamma}$ belongs to the class $R S_{n}^{\gamma}(\alpha, \beta, j)$ and $z$ is real, then

$$
1-\alpha-\sum_{k=j+1}^{\infty}\left(\frac{\gamma+k-1}{\gamma}\right)^{n} \psi_{k} a_{k}(\gamma) z^{k-1} \geq \beta\left|-\sum_{k=j+1}^{\infty}\left(\frac{\gamma+k-1}{\gamma}\right)^{n} \psi_{k} a_{k}(\gamma) z^{k-1}\right|
$$

Letting $z \rightarrow 1$ along the real axis, we obtain the desired inequality

$$
\sum_{k=j+1}^{\infty} \beta\left(\frac{\gamma+k-1}{\gamma}\right)^{n} \psi_{k} a_{k}(\gamma) \leq 1-\alpha-\sum_{k=j+1}^{\infty}\left(\frac{\gamma+k-1}{\gamma}\right)^{n} \psi_{k} a_{k}(\gamma)
$$

which implies that

$$
\sum_{k=j+1}^{\infty}(1+\beta)\left(\frac{\gamma+k-1}{\gamma}\right)^{n}\left|\psi_{k}\right|\left|a_{k}(\gamma)\right| \leq 1-\alpha
$$

Here, it is worthy to note that the function $f(z)^{\gamma}$ given by

$$
f(z)^{\gamma}=z^{\gamma}-\frac{1-\alpha}{(1+\beta)\left(\frac{\gamma+k-1}{\gamma}\right)^{n}\left|\psi_{k}\right|\left|a_{k}(\gamma)\right|} z^{\gamma+j}
$$

is the extremal function.

Corollary 3.6. If the function $f(z)^{\gamma}$ defined by (16) belong to the class $R S_{n}^{\gamma}(\alpha, \beta, j)$, then

$$
a_{k}(\gamma) \leq \frac{1-\alpha}{(1+\beta)\left(\frac{\gamma+k-1}{\gamma}\right)^{n}\left|\psi_{k}\right|}, \quad k \geq j+1
$$

The equality (37) is attained for the function $f(z)^{\gamma}$ given by $(36)$.

\section{Growth and Distortion Properties}

Growth and Distortion properties for function $f(z)^{\gamma}$ in the class $R S_{n}^{\gamma}(\alpha, \beta, j)$ are presented in this section.

Theorem 4.1. Let $f(z)^{\gamma}$ defined by (16) be in the class $R S_{n}^{\gamma}(\alpha, \beta, j)$. Then, for $|z|<r=1, \beta \geq 0$, $-1 \leq \alpha<1$ and $\gamma>0$

$$
r^{\gamma}-\frac{1-\alpha}{(1+\beta)\left(\frac{\gamma+j}{\gamma}\right)^{n}\left|\psi_{j+1}\right|} \leq\left|f(z)^{\gamma}\right| \leq r^{\gamma}+\frac{1-\alpha}{(1+\beta)\left(\frac{\gamma+j}{\gamma}\right)^{n}\left|\psi_{j+1}\right|}
$$


The result (38) is attained for function $f(z)^{\gamma}$ given by (36) for $z= \pm r$.

Proof. Observe from Theorem 3.5 that

$$
(1+\beta)\left(\frac{\gamma+j}{\gamma}\right)^{n}\left|\psi_{j+1}\right| \sum_{k=j+1}^{\infty}\left|a_{k}(\gamma)\right| \leq \sum_{k=j+1}^{\infty}(1+\beta)\left(\frac{\gamma+k-1}{\gamma}\right)^{n}\left|\psi_{j+1}\right|\left|a_{k}(\gamma)\right| \leq 1-\alpha
$$

Thus,

$$
\left|f(z)^{\gamma}\right|=|z|^{\gamma}-\sum_{k=j+1}^{\infty} a_{k}(\gamma)|z|^{\gamma+k-1} \geq r^{\gamma}-r^{\gamma+j} \sum_{k=j+1}^{\infty} a_{k}(\gamma) \geq r^{\gamma}-\frac{1-\alpha}{(1+\beta)\left(\frac{\gamma+j}{\gamma}\right)^{n}\left|\psi_{j+1}\right|} r^{\gamma+j}
$$

Similarly,

$$
\left|f(z)^{\gamma}\right|=|z|^{\gamma}+\sum_{k=j+1}^{\infty} a_{k}(\gamma)|z|^{\gamma+k-1} \leq r^{\gamma}+r^{\gamma+j} \sum_{k=j+1}^{\infty} a_{k}(\gamma) \leq r^{\gamma}+\frac{1-\alpha}{(1+\beta)\left(\frac{\gamma+j}{\gamma}\right)^{n}\left|\psi_{j+1}\right|} r^{\gamma+j} .
$$

From inequalities (40) and (41), we obtain the desired result and this completes the proof of Theorem 4.1.

Theorem 4.2. Let $f(z)^{\gamma}$ defined by (16) be in the class $R S_{n}^{\gamma}(\alpha, \beta, j)$. Then, for $|z|<r=1, \beta \geq 0$, $-1 \leq \alpha<1$ and $\gamma>0$

$$
\gamma r^{\gamma-1}-\frac{(1-\alpha)(\gamma+j)}{(1+\beta)\left(\frac{\gamma+j}{\gamma}\right)^{n}\left|\psi_{j+1}\right|} r^{\gamma+j-1} \leq\left|\left(f(z)^{\gamma}\right)^{\prime}\right| \leq \gamma r^{\gamma-1}+\frac{(1-\alpha)(\gamma+j)}{(1+\beta)\left(\frac{\gamma+j}{\gamma}\right)^{n}\left|\psi_{j+1}\right|} r^{\gamma+j-1}
$$

Proof. The proof is much similar to that of Theorem 4.1.

Letting $n=0, \gamma=1, \beta=0$ and $\alpha=-1$ in Theorem 4.1, Then we obtain the following corollary.

Corollary 4.3. Let $f(z)^{\gamma}$ defined by (16) be in the class $R S_{0}^{1}(-1,0, j)$. Then, for $|z|<r=1, \beta \geq 0$, $-1 \leq \alpha<1$ and $\gamma>0$

$$
r-\frac{2}{\left|\psi_{2}\right|} \leq|f(z)| \leq r+\frac{2}{\left|\psi_{2}\right|}
$$

where $\psi_{k}$ is as earlier defined.

likewise if we let $n=0, \gamma=1, \beta=0$ and $\alpha=-1$ in Theorem 4.2, Then we obtain the following corollary.

Corollary 4.4. Let $f(z)^{\gamma}$ defined by (16) be in the class $R S_{0}^{1}(-1,0, j)$.

$$
1-\frac{4 r}{\left|\psi_{2}\right|} \leq\left|f^{\prime}(z)\right| \leq 1+\frac{4 r}{\left|\psi_{2}\right|}
$$

and $\psi_{k}$ is as earlier defined.

\section{Conclusion}

Ultimately, several authors have studied the Bazilevic class $T_{n}^{\gamma}(\beta)$ from different perspectives while their results authenticated diversely in literatures (see [1,2,8,9] among others). The inclusion of the famous generalized Bessel differential equation in the present work which has a numerous applications in engineering and other fields of science has provided interesting extensions and generalizations of the study of the Bazilevic class $T_{n}^{\gamma}(\beta)$. The results of the extensions and generalizations are detailed in the present article using succinct mathematical approach. 


\section{References}

[1] Babalola, K. O. (2005) Unpublished PhD Thesis. University of Ilorin, Ilorin, Nigeria.

[2] Hamzat, J. O. (2017) Coefficient Bounds for Bazilevic Functions Associated with Modified Sigmoid Function. Asian Research J. Math., 5(3), 1-10.

[3] Hamzat, J. O. and Raji, M. T. (2016) Subordination Conditions for Certain Subclass of Non-Bazilevic Functions in the Open Unit Disk. Int. J. Latest Eng. Tech., Management and Appl. Sci., 1(1), 37-44.

[4] Hamzat, J. O. and Raji, M. T. (2018) Bessel Functions in the Space of $\lambda$-Pseudo-Starlike Functions with Respect to other Points Associated with Modified Sigmoid function. Asian J. Math. Comput. Research, 25(1), 38-49.

[5] Jack, I. S. (1971) Functions Starlike and Convex of Order $\alpha$, J. London Math. Soc., vol.3, 469-474.

[6] Magesh, N. and Prameela, V. (2013) Certain subclasses of uniformly convex functions and corresponding class of starlike functions. Malaya J. Matematik, vol.1, 18-26.

[7] Mondal, S. R. and Swaminathan, A. (2012) Geometric properties of generalized Bessel functions. Bull. Malays. Math. Sci. Soc., 2(35), 179-194.

[8] Oladipo, A. T. and Breaz, D. (2013) A brief study of certain class of Harmonic Functions of Bazilevic Type. ISRN Math. Anal. Article ID 179856, 11 pages.

[9] Opoola, T. O. (1994) On a new subclass of univalent functions. Mathematica, vol.36, no.2, tome (36), 195-200.

[10] Porwal, S. and Ahamad, D. (2015) Connections between certain classes of Hermonic univalent mappings involving generalized Bessel functions. Golf J. Math., 3(4), 98-110.

[11] Shiraishi, H. and Owa, S. (2009) Starlikeness and convexity for analytic functions concerned with Jack's Lemma. Int J. Open Problems in Comput. Sci. Math., 2(1), 37-47.

[12] Singh, S. and Gupta, S. (2009) An extension of the variability of a subclass of univalent functions. J. Ineq. Pure and Appl. Math., vol.10, no.4, article 113, 7 pages. 